\title{
Horizon-T experiment status
}

\author{
Dmitriy Beznosko ${ }^{1, a}$, Rashid Beisembaev ${ }^{2}$, KanatBaigarin ${ }^{1}$, Elena Beisembaeva ${ }^{2}$, Oleg Dalkarov², Vladimi Ryabov ${ }^{2}$, \\ Turlan Sadykov ${ }^{3}$, Sergei Shaulov ${ }^{2}$, Alekse Stepanov ${ }^{2}$, Marina Vildanova ${ }^{2}$, Nikolay Vildanov ${ }^{2}$, and Valeriy Zhukov ${ }^{2}$ \\ 1 Nazarbayev University, Physics Department, Astana, KZ \\ 2 P. N. Lebedev Physical Institute of the Russian Academy of Sciences, Moscow, Russia \\ 3 LLP "Institute of Physics and Technology", Almaty, Kazakhstan
}

\begin{abstract}
Horizon-T is an innovative detector system constructed to study Extensive Air Showers (EAS) in the energy range above $10^{16} \mathrm{eV}$ coming from a wide range of zenith angles $\left(0^{\circ}-85^{\circ}\right)$. The system is located at the Tien Shan High-altitude Science Station of the Lebedev Physical Institute of the Russian Academy of Sciences at $\sim 3340$ meters above sea level. It consists of eight charged particle detection points separated by distances up to one kilometer as well as an optical detector subsystem to measure the Vavilov-Cherenkov light from the EAS.

The time resolution of charged particles and Vavilov-Cherenkov light photons passage of the detector system is a few ns. This level of resolution allows conducting a research of the atmospheric development of individual EAS. This report focuses on a general description of the detector system and the individual sub-systems providing an overview of the operations and latest results.
\end{abstract}

\section{Introduction}

The Tien Shan High-altitude Science Station (TSHSS), of the P. N. Lebedev Physical Institute of the Russian Academy of Sciences, is located at a height of $\sim 3340$ above sea level. The Horizon-T [1] (HT) detector system was constructed there in order to study both the spatial and temporal distribution of the charged particles within the EAS with a time resolution of few ns. This new method allows to collect more data from each detection point (particle density and time of passage) and increase the measurement precision of the EAS parameters and accurately measure the core position even if it falls outside of the detector system area.

HT consists of eight charged particle detection points separated by distances up to one kilometer as well as an optical detector subsystem to view the Vavilov-Cherenkov light from the EAS. The aerial view of the detector system is shown in Fig. 1.

\section{HT detectors}

The HT detector system consists of detectors of several different types: plastic scintillator [2] with MELTS [3] FEU49 model PMT, plastic scintillator with Hamamatsu [4] R7723 PMT assembly, glass with R7723 PMTs and a Vavilov-Cherenkov radiation detector with both FEU49 and Hamamatsu H6527 PMT assemblies.

\subsection{Scintillator detectors}

The Scintillator Detectors (SD) all use polystyrenebased square-shaped cast scintillator with $1 \mathrm{~m}^{2}$ area and $5 \mathrm{~cm}$ thickness. The near periphery SD (detection points

\footnotetext{
a e-mail: dmitriy.beznosko@nu.edu.kz
}

1, 4, 5, 6 and 7) use R7723 PMT assembly as photodetector, and the far periphery (points 2,3 and 8) all use FEU49. This is due to the fact that long cables are used to connect these points to the Data Acquisition system (DAQ) which is located at detection point 1 . The cable calibration [5] shows that signal widening becomes significant thus justifying the use of FEU49 as they have higher gain but wider initial pulse. Three SD, oriented perpendicular to each other in the $\mathrm{x}, \mathrm{y}$ and $\mathrm{z}$ planes, are located at each detection point. The z-plane is parallel to the sky, the $\mathrm{x}$-axis is directed north.

Each SD is calibrated using the signal from minimum ionizing particles (MIP) from the incoming cosmic ray flux. For that, an additional trigger detector consisting of FEU49 and a $15 \mathrm{~cm}$ diameter scintillator is spaced under each SD being tested. Double-coincidence schema is used. The resulting calibration gives the area of a single MIP signal as well as the width of the MIP signal pulse from each SD. Due to analysis specifics of the data, calibration is taken between the 0.1 and 0.9 of the pulse area. This also excludes the baseline noise effects. The area of a MIP signal for the z-plane SD from detection point 1 is shown in Fig. 2. The Landau curve fit gives the most probable value used for the calibration.

We define the front edge of a pulse as the time between 0.1 and 0.5 of the pulse area. With that definition, the pulse front of the SD with R7723 PMT is 5.9 $\pm 1.1 \mathrm{~ns}$ as seen from Fig. 3. The total width is a time between 0.1 and 0.9 of the pulse area and is $18.2 \pm 4.2 \mathrm{~ns}$. Both values are for the z-plane SD from detection point 1 but are typical for any SD with R7723 PMT. Here all uncertainties quoted are the widths of the distributions from the fit and not the errors on the parameter. The uniformity of the response across the SD area is also studied. 


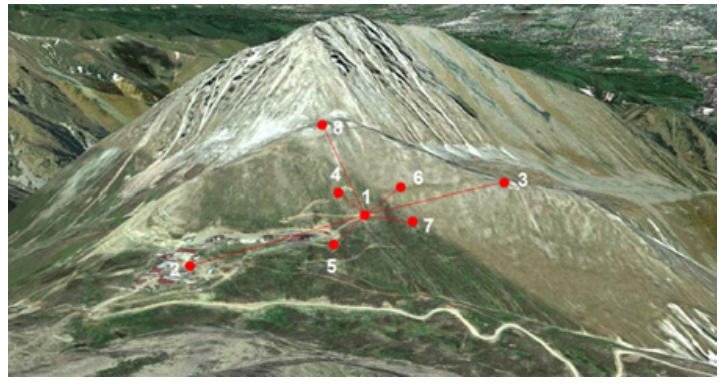

Figure 1. Aerial view of the Horizon-T detector system.

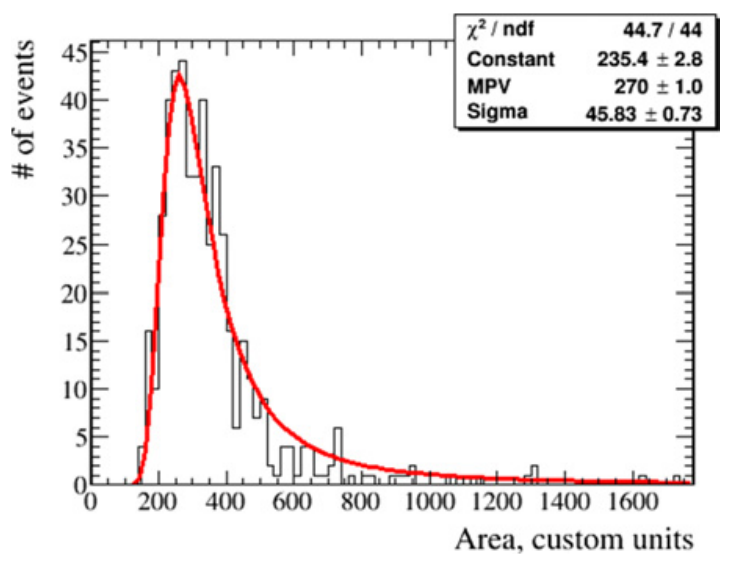

Figure 2. MIP area for SD.

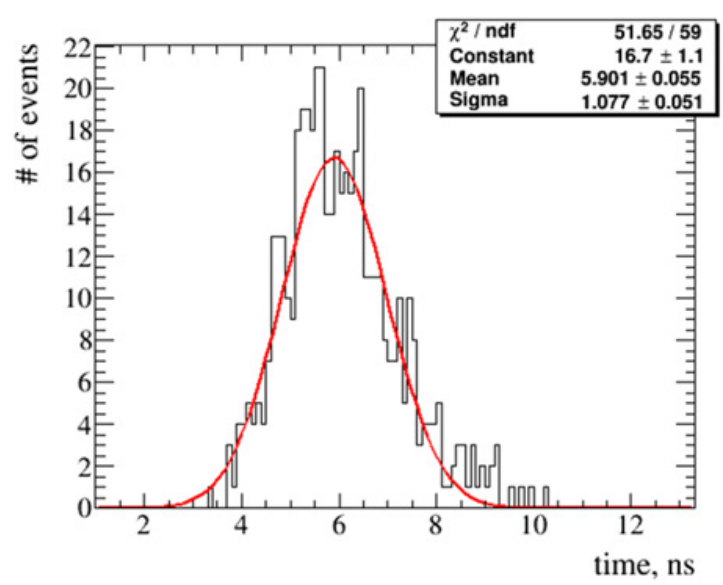

Figure 3. Front edge for SD detector pulse with R7723 PMT.

The SD with the FEU49 also get their MIP response calibrated using exactly the same procedure. From that process, we also get the following typical timings: pulse front $23.2 \pm 5.1 \mathrm{~ns}$ and total width of $102 \pm 29 \mathrm{~ns}$ (the pulse front and the total duration for the FEU49 itself are $15.7 \pm 0.5 \mathrm{~ns}$ and $38.9 \pm 1.4 \mathrm{~ns})$. Data is for the $\mathrm{z}$-plane SD from detection point 2 and includes the clearly seen influence of the long cables used to connect these points to the DAQ.

\subsection{Glass detectors}

At detection points 1, 4, 5, 6 and 7 there are also glassbased detectors (GD) together with the SD. Each GD uses $50 \mathrm{~cm} \times 50 \mathrm{~cm} \times 3 \mathrm{~cm}$ optical glass that is painted white using $\mathrm{TiO}_{2}$ and $\mathrm{R} 7723$ PMT assembly. The reason that only the near periphery is equipped with these detectors is that the very narrow pulse they produce gets widened

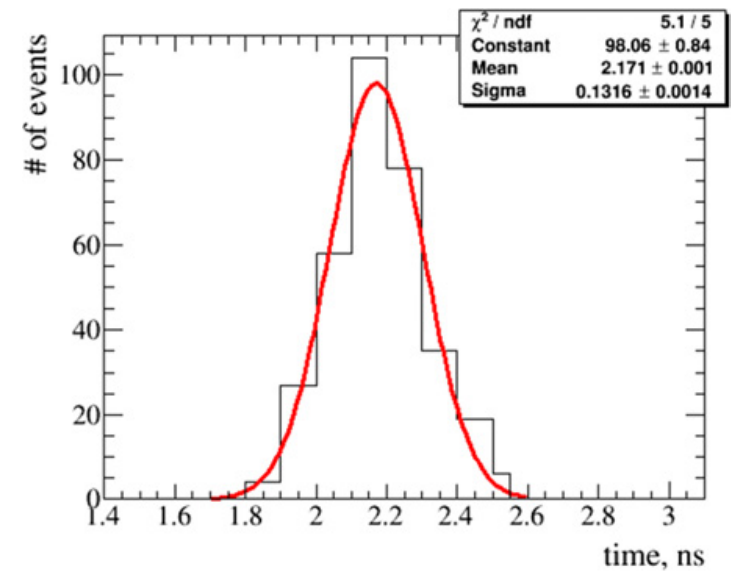

Figure 4. Front edge for GD detector pulse.

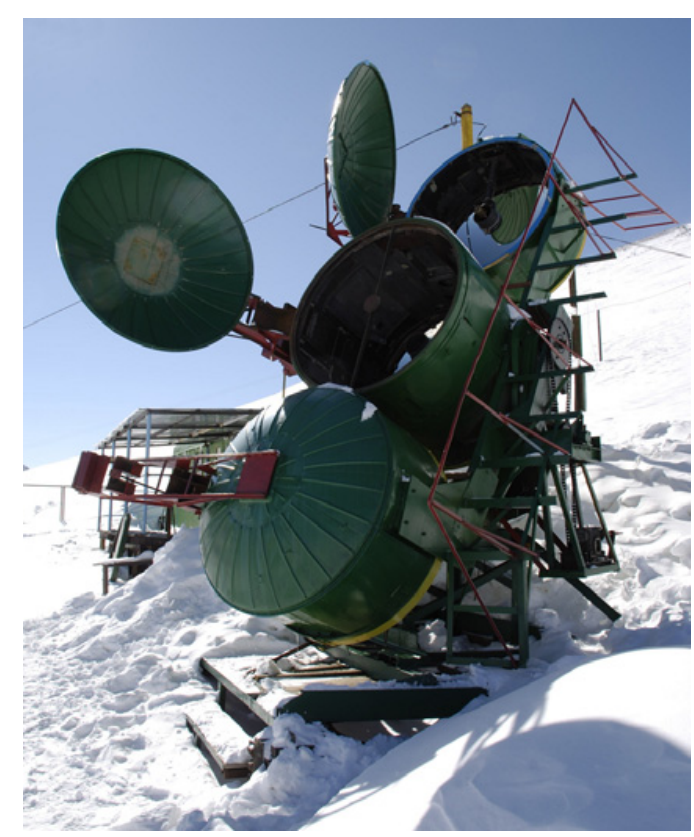

Figure 5. Vavilov-Cherenkov radiation detector.

by the long cable thus diminishing the usefulness of the GL. As measured at detection point 1 , the pulse front is $2.17 \pm 0.13 \mathrm{~ns}$ (see Fig. 4) and the total width is $5.1 \pm 0.7 \mathrm{~ns}$.

\subsection{Vavilov-Cherenkov radiation detector}

Next to detection point 1 there is a Vavilov-Cherenkov radiation detector (VCD), shown in Fig. 5. The VCD consists of three parabolic mirrors of $150 \mathrm{~cm}$ diameter and focal length of $65 \mathrm{~cm}$. They are mounted on the rotating support allowing detection in zenith angle range of $0^{\circ}-80^{\circ}$ and in azimuthal angle range of $0^{\circ}-360^{\circ}$.

A $15 \mathrm{~cm}$ diameter FEU49 and a $15 \mathrm{~cm}$ H6527 PMTs are located in the focal point of each mirror. The field of view of each mirror + PMT is then $\sim 3^{\circ}$.

\section{Data acquisition system and trigger logic}

The DAQ for the HT detector system currently consists of 3 analog to digital converter (ADC) units and in-house written custom software. The units used are the desktop 


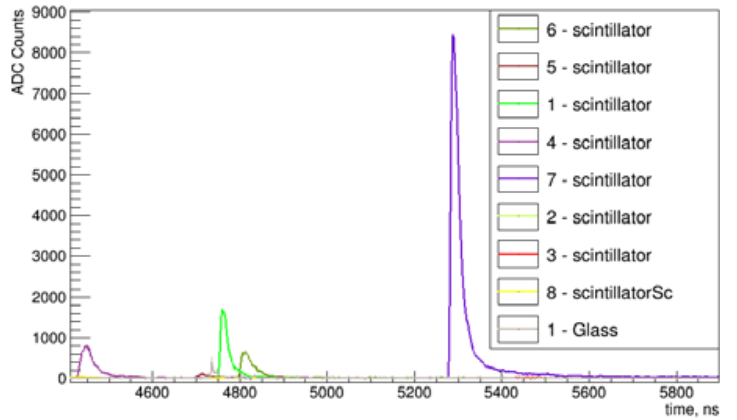

Figure 6. HT sample event.

CAEN [6] DT5730 ADC that do not require a separate crate for operation. They are each read by a computer via USB port.

The multi-threaded DAQ software takes advantage of the multiple CPUs in current computers. One thread is handling data communications, one is for data display, one saves the data as a compressed binary tree format, another controls the process for the failure - resistant operations of the DAQ. The graphical interface and file format are implemented using ROOT [7] framework. Both the internal ADC time stamp and the computer system time are used for best event timing precision.

One of the ADCs is designated as a main board and is used to form a hardware master trigger using double coincidence from detection points 5 and 6 (or 4 and 7). Then the signal is fed via fan-out unit into all three ADCs. The same trigger signal is used for the synchronization pulse that is recorded by the 8th channel of each ADC. The total channel capacity of the DAQ is then 21 channels (with 3 channels used for the sync pulse).

The hardware trigger used is quite relaxed, thus a number of empty events are collected. The offline trigger is applied to all files before any further analysis. Typically we require at least 4 detection points to have a signal in them at the level above the 7 widths of the noise floor, taken as the standard deviation over the first 200 points of each channel that never have any signal in them by design. The resultant rate of EAS registration intensity rate is $\sim 120$ events/day at the HT detector system.

\section{Sample data}

A sample of a typical event detected by the near periphery is shown in Fig. 6. The raw data for each channel is shown, only cable length correction is introduced.

\section{Conclusion}

The Horizon-T detector system is designed for measuring the spatial and temporary structure of incoming EAS with axis in a wide range of zenith angles from $0^{\circ}-80^{\circ}$. HT is located at an altitude of about 3340 meters above sea level centered at geographical coordinates of $43^{\circ} 02^{\prime}$ $49^{\prime \prime} \mathrm{N}$ and $76^{\circ} 56^{\prime} 43^{\prime \prime} \mathrm{E}$. The overall detection rate of EAS starting with primary particle energy at $\sim 10^{16} \mathrm{eV}$ is 120 events/day.

HT has eight charged particle detection points, separated by hundreds of meters. Each point has scintillator detectors, near periphery is also equipped with glass ones.

Detectors are read out by both FEU49 and Hamamatsu R7723 PMTs. Glass detectors with R7723 reach pulse front edge resolution of $\sim 2.2 \mathrm{~ns}$.

The detector system also includes Vavilov-Cherenkov radiation detector that is made from mirrors with FEU49 and H6527 PMTs and has a field of view of each mirror + PMT of $\sim 13^{\circ}$. This detector allows measurements of light from EAS disks in the large range of zenith angles down to $80^{\circ}$.

\section{References}

[1] R.U. Beisembaev et al., arXiv: 1605.05179, (2016)

[2] A Baitenov et al., arXiv:1601.00086, (2016)

[3] MELZ-FEU, 4922-y pr-d, 4c5, Zelenograd, g. Moskva, Russia, 124482 http://www.meltz-feu.ru

[4] Hamamatsu Photonics, 314-5 Shimokanzo, Toyookavillage, Iwatagun, Shizuoka-ken, 438-0193 Japan http://www . hamamatsu. com

[5] T Beremkulov et al., arXiv: 1608.04312, (2016)

[6] CAEN S.p.A. Via della Vetraia, 11, 55049 Viareggio Lucca, Italy http: //www. caen.it

[7] R. Brun and F. Rademakers, Nucl. Instrum. Meth. A 389, 81-86 (1997) 